\title{
PARAMETERIZING GENERALIZED LAGUERRE FUNCTIONS TO COMPUTE THE INVERSE LAPLACE TRANSFORM OF FRACTIONAL ORDER TRANSFER FUNCTIONS
}

\author{
Vilem Karsky \\ Brno University of Technology \\ Department of Control and Instrumentation \\ Technicka 3082/12, Brno \\ Czech Republic \\ xkarsk01@stud.feec.vutbr.cz
}

\begin{abstract}
This article concentrates on using generalized Laguerre functions to compute the inverse Laplace transform of fractional order transfer functions. A novel method for selecting the timescale parameter of generalized Laguerre functions in the operator space is introduced and demonstrated on two systems with fractional order transfer functions.
\end{abstract}

Keywords: Fractional order systems, generalized Laguerre functions, free parameters, inverse Laplace transform

\section{Introduction}

Fractional order transfer functions (FOTFs) have been gaining in popularity recently; however, their use is also associated with substantial complexity of the calculations. Such complexity becomes obvious especially when an inverse Laplace transform (ILT) is to be computed. In study [1], generalized Laguerre functions (GLFs) are interpreted as an instrument suitable for the given task; GLFs nevertheless have free parameters, and this property affects the approximation speed. Reflecting the drawback, the present paper proposes a technique to select the "good" free parameters.

\section{Options}

\subsection{Inverse Laplace Transform using Mittag-Leffler Functions}

We can calculate an ILT classically, utilizing the formula from book [2]

$$
\mathscr{L}\left\{t^{\alpha k+\beta-1} E_{\alpha, \beta}^{(k)}\left( \pm a t^{\alpha}\right)\right\}=\frac{k ! s^{\alpha-\beta}}{\left(s^{\alpha} \mp a\right)^{k+1}},
$$

where $E_{\alpha, \beta}^{(k)}\left( \pm a t^{\alpha}\right)$ are Mittag-Leffler functions (MLFs), defined by

$$
E_{\alpha, \beta}(z)=\sum_{k=0}^{\infty} \frac{z^{k}}{\Gamma(\alpha k+\beta)} .
$$

As indicated above, MLFs embody the generalization of an exponential function in the form of an infinite series. Converging the series is a very slow process involving problematic numerical stability for the higher orders of this series, Fig. 1; the first 120 terms of the series were used in the procedure. 


\subsection{Inverse Laplace Transform using GLFs}

GLFs are defined according to [1] by

$$
l_{n}^{\alpha}(2 \lambda t)=\sqrt{2 \lambda t} \mathrm{e}^{-\lambda t} L_{n}^{\alpha}(2 \lambda t)
$$

where $L_{n}^{\alpha}(x)$ are the generalized Laguerre polynomials, characterized in [3] as

$$
L_{n}^{\alpha}(x)=\frac{\mathrm{e}^{x} x^{-\alpha}}{n !} \frac{\mathrm{d}^{\mathrm{n}}}{\mathrm{d} x^{n}}\left(x^{n+\alpha} \mathrm{e}^{-x}\right) .
$$

The referenced article [1] proposes a manner of using GLFs to compute an ILT and outlines a simpler approach to calculating the spectrum coefficients in the GLF base when the free parameter $\alpha=1$. The formula for the spectrum coefficients reads

$$
c_{i}^{1}=\frac{-\sqrt{2 \lambda}}{(i+1) !}\left[\frac{\mathrm{d}^{i+1}}{\mathrm{~d} z^{i+1}} F(z)\right]_{z=0},
$$

where $F(z)$ is our transfer function after being subjected to the bilinear transform

$$
s=\lambda \frac{1+z}{1-z} .
$$

But, at this stage, there still remains one free parameter, namely, the timescale $\lambda$, which affects the convergence rate. With a good timescale, the method converges considerably faster than the MLF-based solution.

\subsection{Parameterizing GLFs in the Time Space}

The authors of source [4] designed a technique for choosing the "good" values in the free parameters of GLFs; these parameters, despite being "good", are nevertheless not optimal. To calculate them, we need to establish the moments first; we then have

$$
\begin{aligned}
m_{-1} & =\left\langle f(x), x^{-1} f(x)\right\rangle, & & m_{1}=\langle f(x), x f(x)\rangle, \\
m_{0} & =\langle f(x), f(x)\rangle, & & m_{2}=\left\langle f^{\prime}(x), x f^{\prime}(x)\right\rangle,
\end{aligned}
$$

where $\langle f(x), g(x)\rangle$ denotes the scalar product. From the moments, it is possible to calculate the free parameters' "good" values, using the formulae

$$
\begin{aligned}
2 \lambda & =2 \sqrt{\frac{m_{-1} m_{2}}{\left|m_{1} m_{-1}-m_{0}^{2}\right|}}, \\
\alpha & =\frac{m_{0}}{m_{-1}} 2 \lambda .
\end{aligned}
$$

\section{$3 \quad$ Parameterizing GLFs in the Operator Space}

The impulse response of a stable system quickly leads to a zero. As is shown in Fig. 1, the numerically unstable part of the response given by MLFs is almost a zero in the real domain. This approach to computing the free parameters exploits the assumption of a stable transfer function and a response leading to a zero at a quick pace.

When computing an ILT, we first utilize MLFs (section 2.1). From the impulse response, only the initial numerically stable part will then be used, with the remaining portions being zero. Thus, we can compute the moments and timescale $\lambda$ according to section 2.3; the final impulse response is computable as outlined in section 2.2, exploiting a good timescale.

The method finds use only in stable systems as it requires an impulse response that quickly nears zero in the manner characterized above.

\section{Examples}

The technique is demonstrated below on two systems with a fractional order transfer function. 


\subsection{First Transfer Function}

The first system is described by means of the transfer function

$$
F(s)=\frac{1}{s^{1.3}+7}
$$

When calculating an inverse Laplace transform via (1), we get the impulse response

$$
g(t)=\mathscr{L}^{-1}\left\{\frac{1}{s^{1.3}+7}\right\}=t^{0.3} E_{1.3,1.3}\left(-7 t^{1.3}\right)=\sum_{k=0}^{\infty} \frac{(-7)^{k} t^{1.3 k+0.3}}{\Gamma(1.3 k+1.3)}
$$

This response of the initial 120 terms is displayed in Fig. 1.

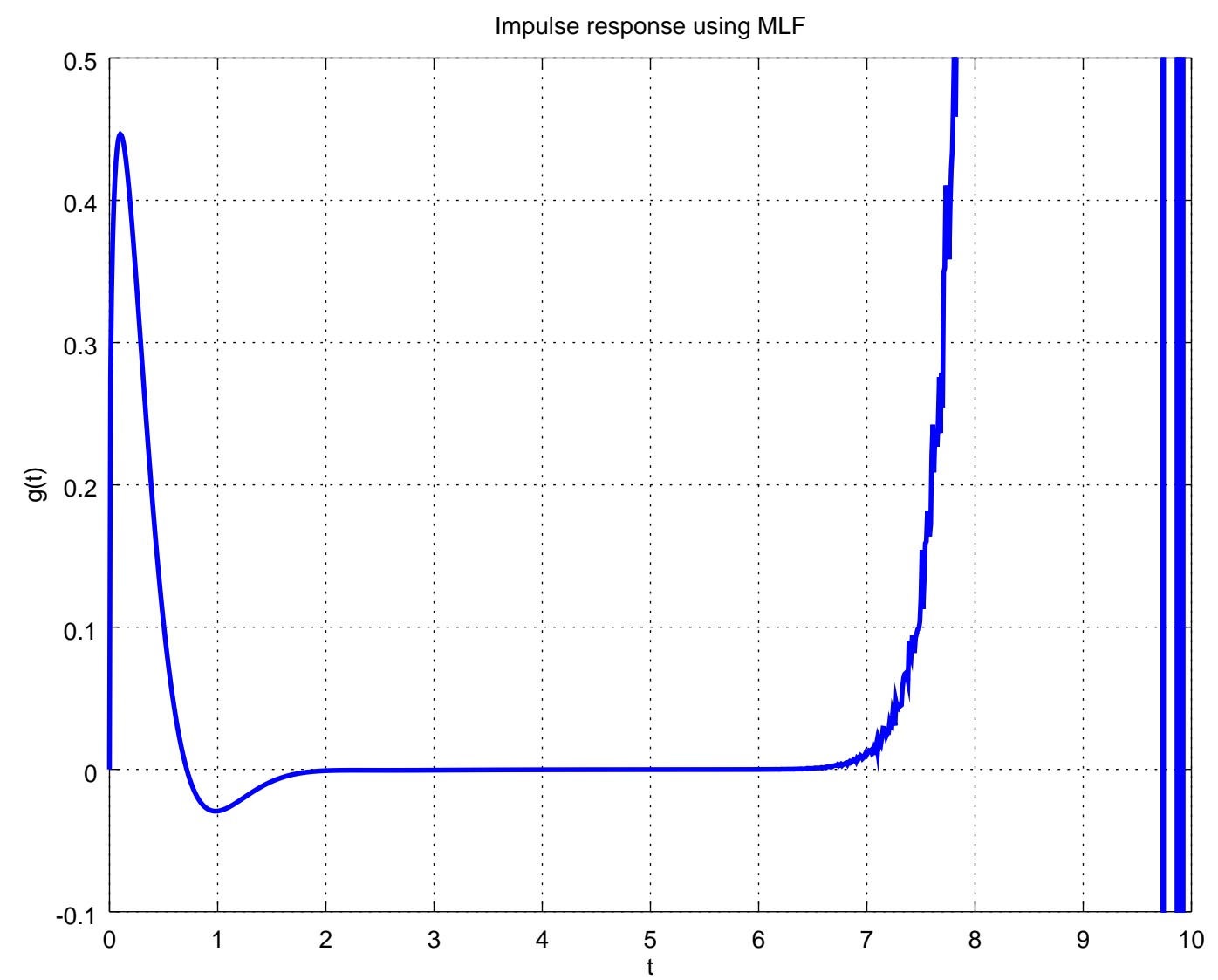

Figure 1: An Inverse Laplace Transform using MLFs.

From this impulse response, we calculated the moments and timescale $\lambda$ according to section 2.3 ; the results are presented in Table 1.

Table 1: The moments and timescale for the first system.

\begin{tabular}{|c|c|c|c|c|}
\hline$m_{-1}$ & $m_{0}$ & $m_{1}$ & $m_{2}$ & $\lambda$ \\
\hline 0.6040 & 0.0538 & 0.0100 & 0.1454 & 5.2751 \\
\hline
\end{tabular}

Computing the ILT using GLFs, we obtain the spectrum coefficients shown in Table 2.

Table 2: The spectrum of the first system in the GLF base.

\begin{tabular}{|c|c|c|c|c|c|c|c|}
\hline$c_{0}$ & $c_{1}$ & $c_{2}$ & $c_{3}$ & $c_{4}$ & $c_{5}$ & $c_{6}$ & $c_{7}$ \\
\hline 0.2981 & -0.0416 & -0.0627 & 0.0183 & 0.0013 & -0.0029 & -0.0014 & 0.0027 \\
\hline
\end{tabular}


The two approaches are compared in Fig. 2.

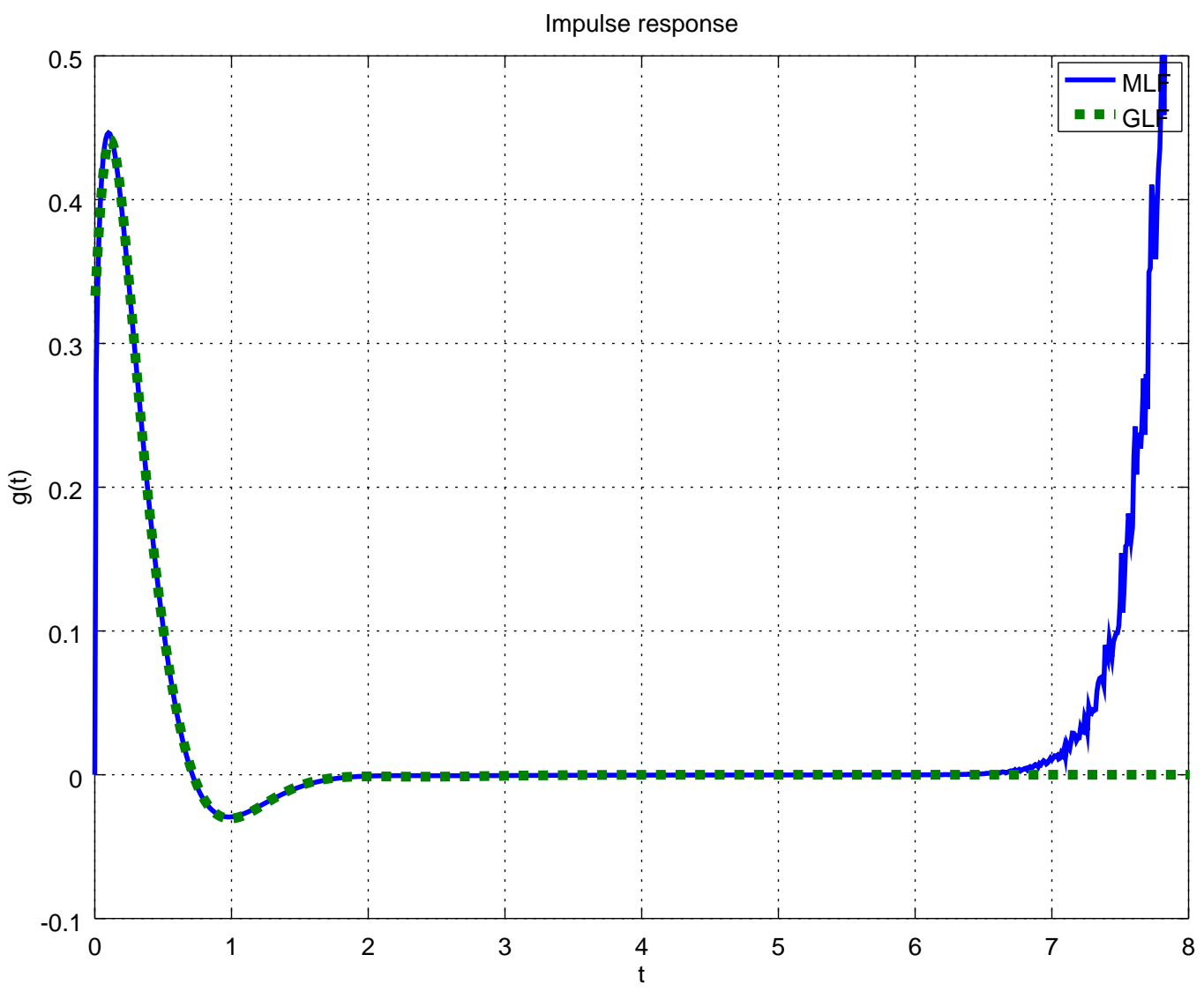

Figure 2: A comparison of the methods.

\subsection{Second Transfer Function}

The second system is characterized through the transfer function

$$
F(s)=\frac{1}{s^{1.4}+s^{0.7}+1}=\frac{\mathrm{j} \frac{\sqrt{3}}{3}}{s^{0.7}+\frac{1+\mathrm{j} \sqrt{3}}{2}}-\frac{\mathrm{j} \frac{\sqrt{3}}{3}}{s^{0.7}+\frac{1-\mathrm{j} \sqrt{3}}{2}} .
$$

Here, when calculating an inverse Laplace transform via (1), we get the impulse response

$$
g(t)=\mathscr{L}^{-1}\left\{\frac{1}{s^{1.4}+s^{0.7}+1}\right\}=\mathrm{j} \frac{t^{-0.3}}{\sqrt{3}}\left[E_{0.7,0.7}\left(-\frac{1+\mathrm{j} \sqrt{3}}{2} t^{0.7}\right)-E_{0.7,0.7}\left(-\frac{1-\mathrm{j} \sqrt{3}}{2} t^{0.7}\right)\right] .
$$

The response for the initial 60 terms is displayed in Fig. 3. 


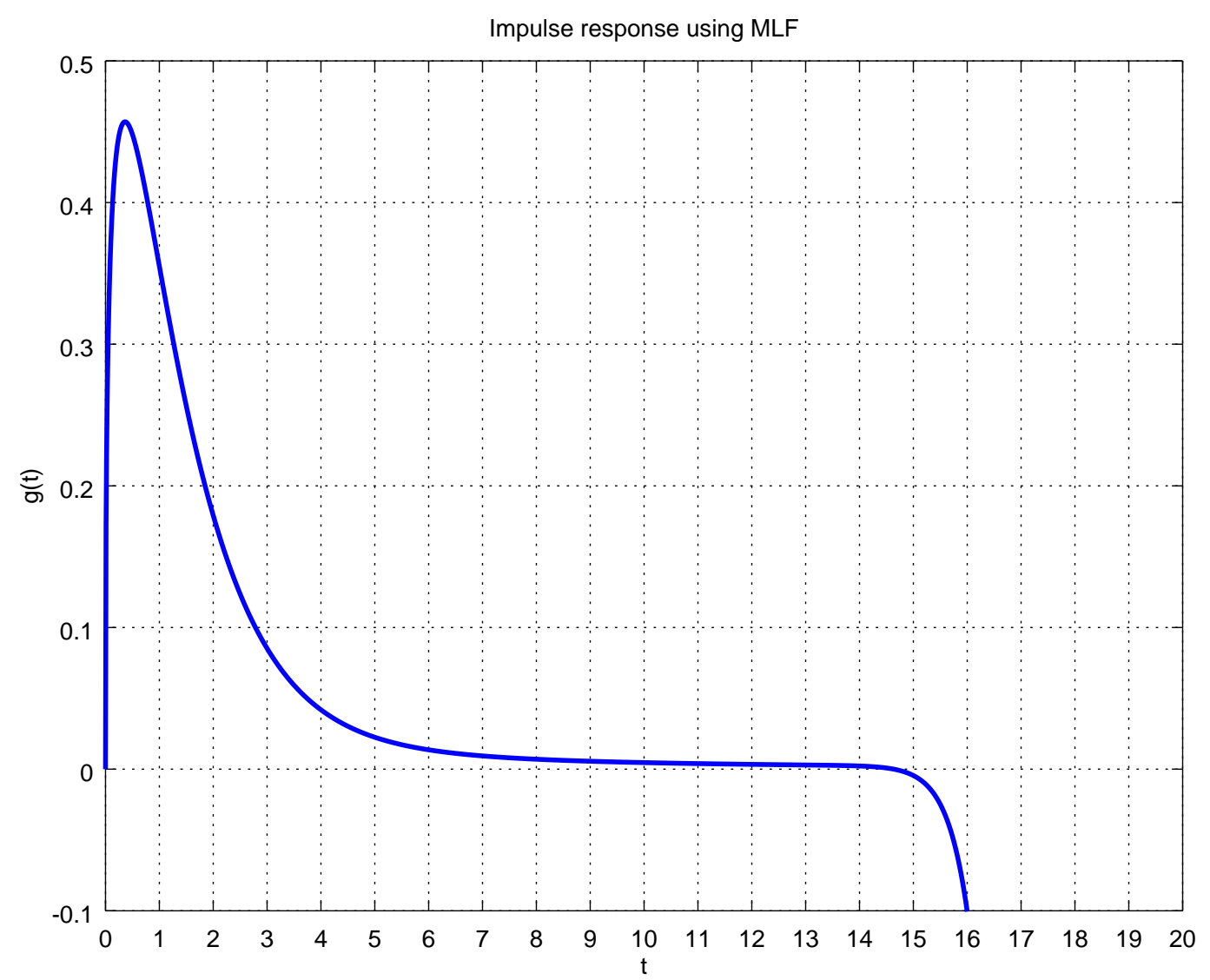

Figure 3: An Inverse Laplace Transform using MLFs.

From this impulse response, we calculated the moments and timescale $\lambda$ according to section 2.3; the results are presented in Table 3 .

Table 3: The moments and timescale for the second system.

\begin{tabular}{|c|c|c|c|c|}
\hline$m_{-1}$ & $m_{0}$ & $m_{1}$ & $m_{2}$ & $\lambda$ \\
\hline 0.6797 & 0.1415 & 0.0713 & 0.1180 & 1.6783 \\
\hline
\end{tabular}

Computing the ILT using GLFs, we obtain the spectrum coefficients summarized in Table 4.

Table 4: The spectrum of the second system in the GLF base.

\begin{tabular}{|c|c|c|c|c|c|c|c|}
\hline$c_{0}$ & $c_{1}$ & $c_{2}$ & $c_{3}$ & $c_{4}$ & $c_{5}$ & $c_{6}$ & $c_{7}$ \\
\hline 0.7045 & -0.3605 & 0.1143 & -0.0569 & 0.0248 & -0.02004 & 0.0127 & -0.0074 \\
\hline
\end{tabular}

The approaches are compared in Fig. 4. 


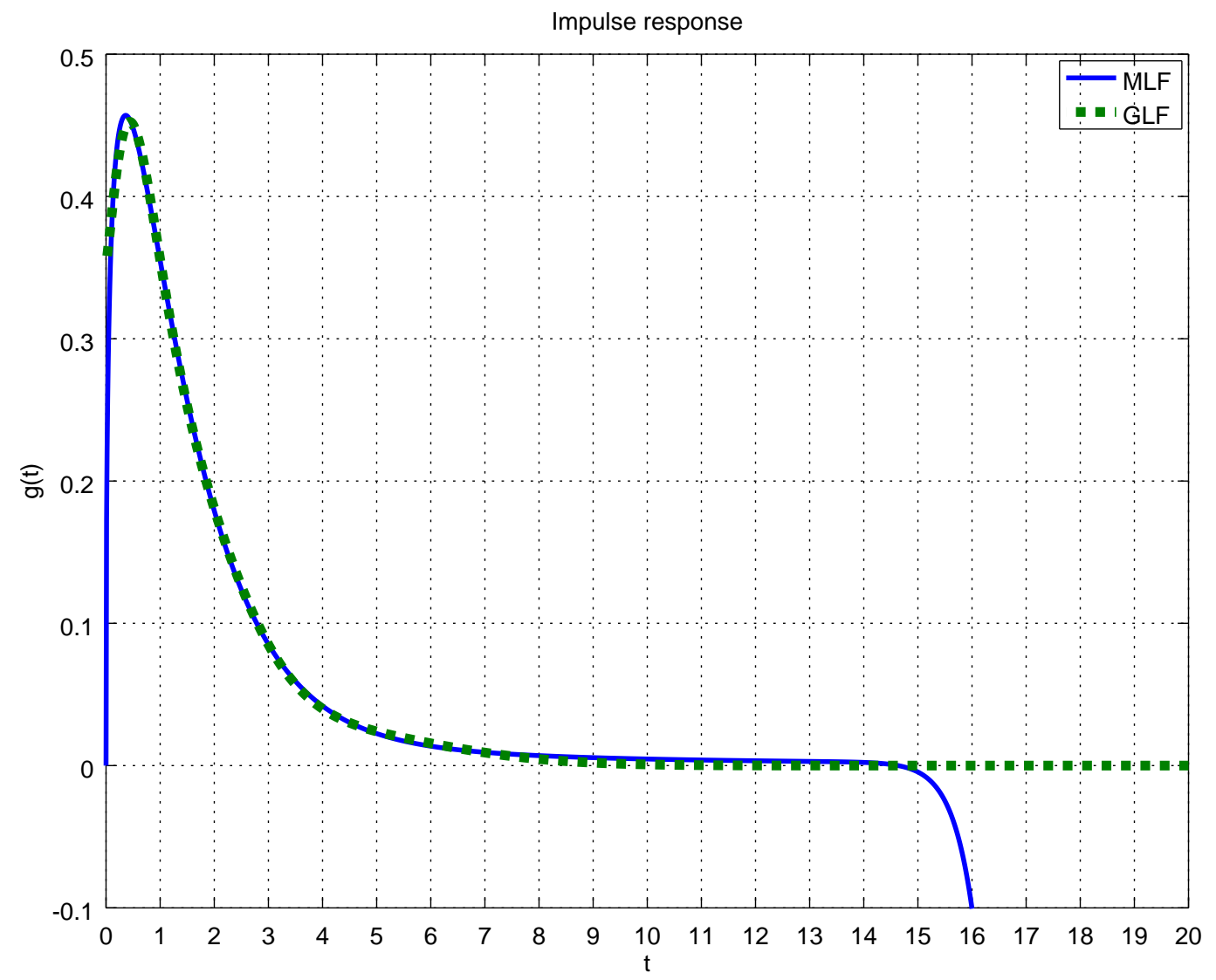

Figure 4: A comparison of the methods.

\section{Conclusion}

The article [1] shows that GLFs are suitable for computation of an inverse Lapalce tansform and in this paper a method how to choose values of free parametrs for GLF is shown. It follows from the analysis that the proposed method of choosing free parameters for generalized Laguerre functions is efficiently applicable in regular conditions but does not perform well with unstable systems.

Acknowledgement: The completion of this paper was made possible by grant No. FEKT-S-17-4234 - "Industry 4.0 in automation and cybernetics" financially supported by the internal science fund of Brno University of Technology.

\section{References}

[1] Mione, G.: Inverting fractional order transfer functions through Laguerre approximation. Systems and Control Letters, 52(5), 387-393 (2004).

[2] Podlubny, I.: Fractional differential equations: an introduction to fractional derivatives, fractional differential equations, to methods of their solution and some of their applications, Mathematics in science and engineering, vol. 198. Academic Press, San Diego, California (1999)

[3] Associated Laguerre Polynomial. Wolfram Math World http://mathworld.wolfram.com/AssociatedLaguerrePolynomial.html (2017). [Online; accessed 17-May$2018]$

[4] Belt, H.J.W., den Brinker, A.C.: Optimal parametrization of truncated generalized Laguerre series. 1997 IEEE International Conference on Acoustics, Speech, and Signal Processing (1997). DOI 10.1109/icassp.1997.604708 